\title{
Ballistic Impact Performance of SiC Ceramic-Dyneema Fiber Composite Materials
}

\author{
Kai-Kuang Wu, ${ }^{1}$ Yu-Liang Chen, ${ }^{2,3}$ Jau-Nan Yeh $\mathbb{D D}^{2,3}$ Wei-Lun Chen, ${ }^{4}$ and Chia-Shih Lin ${ }^{5}$ \\ ${ }^{1}$ Army Aviation Depot, Ministry of National Defense, Taipei, Taiwan \\ ${ }^{2}$ Department of Power Vehicle and Systems Engineering, Chung-Cheng Institute of Technology, National Defense University, \\ Taoyuan, Taiwan \\ ${ }^{3}$ System Engineering and Technology Program, National Chiao Tung University, Hsinchu, Taiwan \\ ${ }^{4}$ Army Second Regional Support Command, Ministry of National Defense, Taipei, Taiwan \\ ${ }^{5}$ National Chung-Shan Institute of Science and Technology, Taoyuan City, Taiwan
}

Correspondence should be addressed to Jau-Nan Yeh; c31000403@gmail.com

Received 24 July 2019; Revised 12 December 2019; Accepted 24 December 2019; Published 22 January 2020

Academic Editor: Fuat Kara

Copyright $(92020$ Kai-Kuang Wu et al. This is an open access article distributed under the Creative Commons Attribution License, which permits unrestricted use, distribution, and reproduction in any medium, provided the original work is properly cited.

This study investigated the ballistic resistance of a composite target plate fabricated by combining SiC ceramic with the Dyneema fiber. To achieve a light-weight target plate that conforms to the US National Institute of Justice level four (NIJ IV) standards, minimal areal density analysis was conducted to obtain the optimal SiC ceramic-Dyneema fiber thickness combination. This study used energy absorption to analyze the ballistic resistance of the target plates. To drastically reduce experimental costs, most of this work employed ANSYS/LS-DYNA software to conduct finite element numerical simulations. First, ballistic experiments that conformed to NIJ IV standards were conducted to verify the simulation parameter configurations. Subsequently, the correlation function of the relationship between the combined thickness of the composite material and its ballistic resistance was determined through the experimental design, which effectively reduced the simulation analysis time. According to simulation experiments and regression analysis, the equation for the relationship between the combined thickness of the composite material and its ballistic resistance was $E_{A}\left(h_{c}, h_{f}\right)=-6276.5+500.6 h_{c}+1512.6 h_{f}+30.7 h_{c} h_{f}-8.1 h_{c}^{2}-113.6 h_{f}^{2}$, though there were limitations to its application. From the numerical analysis results, $8.1940 \mathrm{~mm} \mathrm{SiC} \mathrm{ceramic} \mathrm{and} 6.9637 \mathrm{~mm}$ Dyneema fiber were determined to constitute the optimal thickness combination for a composite that features a minimal areal density and which conforms to NIJ IV standards. The combination was verified to be consistent with the numerical simulation analysis results.

\section{Introduction}

Protective measures on battlefields commonly have a wide range of applications for personnel, vehicles, ships, planes, and buildings. The latest protection technologies are generally used on tanks (armored vehicles) and protective clothing. The continuous enhancement of weapons with the progress of science and technology required the constant development of protective materials and structures. The most widely applied protective materials are currently ceramic composites, which use ceramic as front plates and fiber, metal, or other tough materials as the back plate. This combination is called the ceramic composite (Figure 1).
Ceramic is extremely hard and wear-resistant, which enables the passivation, erosion, and shattering of projectiles, increasing the impacted area between projectiles and armors and dissipating impact load. Furthermore, shattered ceramics cause mutual erosion with projectiles, producing lateral and reverse impact flow and preventing further penetration [1]. Fiber has greater tenacity, specific strength, specific modulus, fatigue resistance, wear resistance, and shock absorption compared with the metal. When used as the back plate material for ceramic composite bulletproof target plates, fibers can delay tensile failure in ceramics, causing projectiles to fully erode the target plate and dissipate kinetic energies, providing support and absorbing the kinetic energy of projectiles and ceramic shards [2]. 


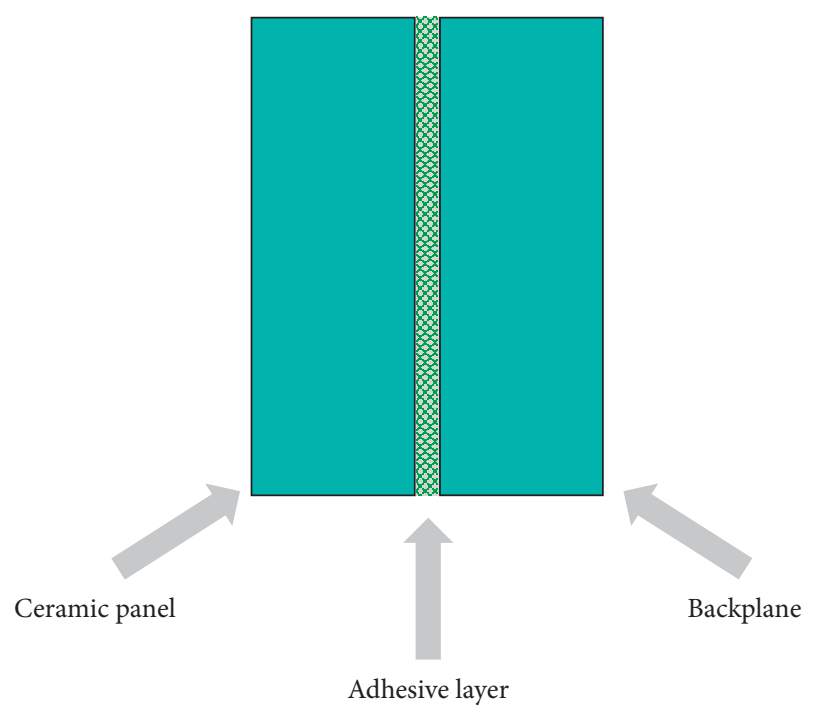

Figure 1: Schematic diagram of the ceramic composite.

Therefore, composite materials that combine ceramic and fiber are currently the most widely applied bullet-resistant material. The results of a review of the literature on ceramic composites and ballistic protection were as follows.

Reviewing studies on the ballistic resistance of ceramic composites before 2008, Hou et al. [3] concluded that findings on the effects of the penetration resistance of ceramic composites had been inconsistent. He proposed that future research should shift from the application of claymetal composites to clay-fiber composites.

In a study on the ballistic resistance of ceramic composite target plates that combined ceramic $\left(\mathrm{Al}_{2} \mathrm{O}_{3}\right)$ with different materials such as steel, aluminum, and spectra fiber as the back plate, Sherman [4] concluded that the ceramic plate was the main factor in resisting bullet penetration.

Du et al. [5] analyzed the ballistic resistance of an $\mathrm{Al}_{2} \mathrm{O}_{3}$ Kevlar fiber composite target plate using the modified hydrodynamics of Alekseevsky-Tate, maximum strain failure theory, and the law of conservation of energy. Du discerned a three-phase mode for the process of shooting a bullet at a target plate and established mathematical equations to present the energy loss in each phase and estimate the ballistic limit $\left(\mathrm{V}_{50}\right)$ of the target plates. After conducting firing tests using $7.62 \mathrm{~mm}$ armor-piercing (AP) incendiary ammunition, Du concluded that increasing the thickness or surface density of the target plate can improve its ballistic resistance, with ceramic plates showing more significant improvements than fiber plates. In addition, increasing the length-diameter ratio of the bullets greatly decreased the ballistic limit $\left(\mathrm{V}_{50}\right)$ of the target plates.

In a study involving ceramic composite target plates with a combination of fiber layer-ceramic plate-fiber layer-aluminum alloy, Li et al. [6] conducted a qualitative analysis on the ballistic resistance of the plates using theoretical models such as stress wave, restraint mechanism, back-force effect, and shock absorption. The results indicated that to improve ballistic resistance, the transmitted wave intensity had to be decreased and the reflected wave intensity had to be increased. This caused the edge of the shell crater to collapse and reduced its perforation size, hindering bullet movement because of the increased thickness of the fabric layer.

Hsieh et al. [7] investigated whether the addition of ceramic plates to aromatic polyamide fabric would alter the fabric's ballistic resistance. Their results indicated that $6 \mathrm{~mm}$ ceramic plates were able to effectively disperse the impact energy of bullets, and the addition of ceramic plates was able to increase the bullet attrition rate.

Chen et al. [8] studied the resistance of ceramic-low carbon steel composite target plates to the impact of $7.62 \mathrm{~mm}$ AP ammunition. The study employed a numerical simulation that combined finite element and smoothed particle hydrodynamics methods, whereby a mathematical relation of ballistic limit $\left(\mathrm{V}_{50}\right)$ constituting the ratio between the thickness and surface density of ceramics and low carbon steel was established. This relation was applicable to the ballistic limit $\left(\mathrm{V}_{50}\right)$ of $400 \mathrm{~m} / \mathrm{s}-800 \mathrm{~m} / \mathrm{s}$ and a ceramic-low carbon steel thickness ratio of 1.5-6.0.

Ömer et al. [9] studied a GFRP composite material milled to experimentally minimize the damages on the machined surfaces, using two, three, and four flute end mills at different combinations of cutting parameters. Experimental results showed that the damage factor increased with increasing cutting speed and feed rate; on the contrary, it was found that the damage factor decreased with increasing depth of the cut and number of the flutes. In addition, analysis of variance (ANOVA) results clearly revealed that the feed rate was the most influential parameter affecting the damage factor in end milling of GFRP composites.

Erkan et al. [10] studied a series of machining operations done for data collection by varying the flute number, feed rate, depth of cut, and cutting speed. The relationship between the cutting parameters of end milling operations and the surface roughness of the machined surface was studied. For the analysis of the data and selection of the optimal cutting parameters, Taguchi method and genetically optimized neural network systems (GONNs) were used.

Gökhan and Ömer [11] indicated that it is possible to improve the whole quality by reducing the delamination which occurs in the drilling of carbon fiber-reinforced plastic (CFRP) composite plates. For the study, carried out under dry machining conditions, the parameters included three different cutting speeds, three different feed rates, and drill geometry involving three different point angles.

This study conducted finite element method numerical simulations using ANSYS/LS-DYNA software to drastically lower live firing costs and simultaneously reduce the number of experiments using the factor combination of the experimental design. In addition, the study used regression analysis to obtain empirical functions and effectively reduce simulation time.

The present study used ballistic-resistant composite target plates composed of silicon carbide ceramic ( $\mathrm{SiC})$ and Dyneema fiber, analyzing their ballistic resistance through energy modes. Ballistic resistance was defined as the kinetic energy difference and the absorptive power of the ceramic composite plate when the bullet penetrated it. Ballistic experiments conforming to the US National Institute of Justice 
level four (NIJ IV) standards were conducted to verify the simulation parameters; factor combinations were planned using the experimental design. Further data regression was conducted to obtain the correlation function between the thickness combination of the ceramic composite ballisticresistant plate and its ballistic resistance. The optimal combined thickness of ceramic and fiber for a light-weight, bullet-resistant ceramic composite target plate with minimal surface density was achieved through the derivation of the aforementioned correlation function. Finally, the optimal combination was verified through ballistic experiments and simulations.

\section{Ballistic Experiments}

2.1. Definition of Ballistic Resistance. To acquire test data that enabled calculation and analysis, a plate penetration experiment was conducted, and the residual velocity of the bullet was determined, from which the kinetic energy difference was obtained. Thus, the study defined ballistic resistance as the kinetic energy difference of the bullet when it penetrated the target plate, i.e., the absorptive energy of the plate (equation (1)). A higher absorptive energy meant the plate possessed a higher ballistic resistance. Because it was difficult to collect the bullets that had penetrated the plates during the ballistic experiments, the mass of the bullet was assumed to remain constant throughout the test:

$$
E_{A}=\frac{1}{2} m v_{i}^{2}-\frac{1}{2} m v_{r}^{2}
$$

where $E_{A}(J)$ represents the ballistic resistance of the plates; $m(\mathrm{~kg})$ represents the bullet weight; $v_{i}(\mathrm{~m} / \mathrm{s})$ represents the initial velocity of the bullet; and $v_{r}(\mathrm{~m} / \mathrm{s})$ represents the residual velocity of the bullet after it penetrated the plate.

2.2. Target Plate Fabrication. The ballistic-resistant ceramic composite target plate mainly consisted of silicon $\mathrm{SiC}$ ceramic and a Dyneema (unidirectional structure) fiber back plate. The ceramic plate was hexagonal with side lengths of $60 \mathrm{~mm}$ each, fabricated by sintering $\mathrm{SiC}$ ceramic powder under atmospheric pressure at a temperature of $2050^{\circ} \mathrm{C}$. A $200 \mathrm{~mm} \times 200 \mathrm{~mm}$ piece of Dyneema fabric was cut and hotpressed into the plate at a temperature of $115^{\circ} \mathrm{C}$. The ceramic and fiber plates were then bound together using epoxy resin and compressed under a $10 \mathrm{~kg}$ weight for $24 \mathrm{~h}$ to ensure they were firmly joined.

2.3. Ballistic Test Arrangement. This study conducted ballistic test in accordance with the NIJ IV standard, using $0.30^{\prime \prime}$ AP ammunitions, with the amount of gunpowder adjusted to achieve an initial velocity of $868 \mathrm{~m} / \mathrm{s} \pm 15 \mathrm{~m} / \mathrm{s}$. Photoelectric screens were used to measure the velocity of the bullet, whereby two screens were placed $1 \mathrm{~m}$ apart to separately measure the initial and residual velocities of the bullet by transmitting the photoelectric signals to the oscilloscope. The smallest measured scale was $10^{-6}$ sec. Figure 2 shows a schematic diagram of the ballistic experiments.

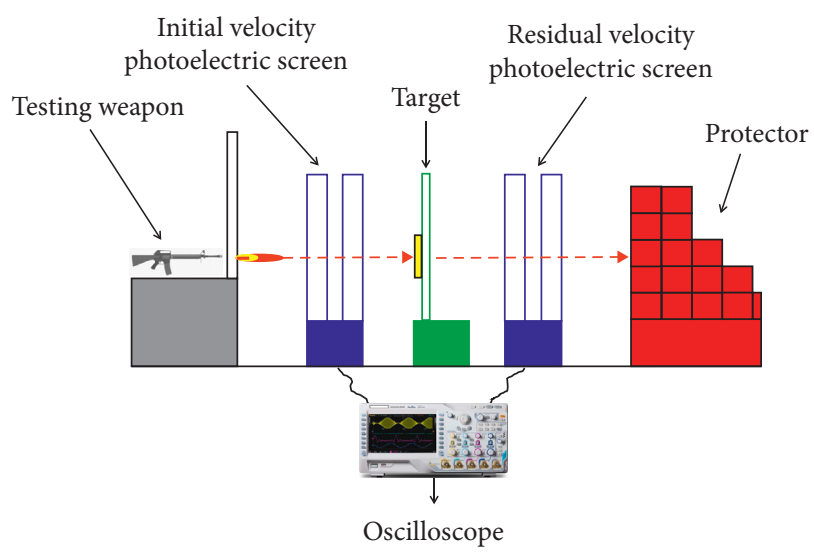

FIGURE 2: Schematic diagram of ballistic test.

2.4. Ballistic Test Results. To reduce the influences of factors such as temperature and humidity on the results, the experiment was completed within the same day and conducted in triplicate to ensure its accuracy. To verify the parameter settings of the finite element numerical simulation, $6 \mathrm{~mm}$ silicon carbide ceramic and a $6 \mathrm{~mm}$ Dyneema fiber were bound and used as the ceramic composite target plate, which was then used to conduct the target plate penetration experiment under the NIJ IV standard using 0.30" AP ammunitions. The initial and residual velocities of each bullet after it penetrated the plate were measured to calculate the absorptive energy of the plate, as shown in Table 1. The bullet mass $(m)$ was measured as $10.6 \mathrm{~g}$ and was held constant throughout the experiment.

Despite minor variations in the initial velocity, it still fell within the range of NIJ IV standards with a difference of less than $1.263 \%$, evidence that this experiment was well controlled. The measured absorptive energy of the target plate also varied, but the difference was less than $0.164 \%$, indicating the stability and consistency of this experiment. Therefore, the influence of the initial velocity difference of the bullet on the absorptive energy of the target plate could be disregarded.

The second set of data from the ballistic test was compared with the analysis results to verify the finite element numerical simulation. To completely eliminate the influence of the initial velocity difference of the bullet on the absorptive energy of the target plate, the initial velocity was set as $875 \mathrm{~m} / \mathrm{s}$ during the finite element simulation analysis.

\section{Finite Element Numerical Simulation Analysis}

3.1. ANSYS/LS-DYNA Finite Element Simulation Software. The LS-DYNA simulation software facilitates numerical analysis on high-speed impact and penetration experiments. It is among the most powerful software programs involving dynamic finite element analysis and is widely applied in the defense and civil sectors. The software mainly operates using an explicit integral algorithm, with a minor integral algorithm acting as a supplementary mode. It also has three numerical description methods: Lagrangian, Eulerian, and 
TABLe 1: Correctness of experimental.

\begin{tabular}{lccc}
\hline No. & $v_{i}(\mathrm{~m} / \mathrm{s})$ & $v_{r}(\mathrm{~m} / \mathrm{s})$ & Absorptive energy of the target plate $(\mathrm{J})$ \\
\hline 1 & 871 & 536 & 2,498 \\
2 & $\mathbf{8 7 5}$ & $\mathbf{5 4 2}$ & $\mathbf{2 , 5 0 1}$ \\
3 & 882 & 553 & 2,502 \\
\hline
\end{tabular}

arbitrary Lagrangian-Eulerian and is suitable for static load analysis as well as studying brief or instantaneous dynamic behaviors with short action times, large deformations, nonlinear structural impacts, and complicated contact impacts [12].

\subsection{Procedure of the Finite Element Method Numerical} Simulation. The finite element method numerical simulation procedure in this study was divided into two parts. First, the ANSYS program was used as a preprocessor to establish high-speed impact numerical analysis models for the bullet and the target plate. This included model drawing, meshing, defining material properties, selecting material models, and configuring the contact mode, as well as control parameters and boundary conditions, which in turn produced the keyword file.

Next, the keyword file was input into the LS-DYNA software solver to determine the solution and was further processed by the LS-PrePost to analyze the penetration and destruction mode of the target plate, as well as evaluate the residual velocity of the bullet.

3.3. Material Model Construction. The bullet model was based on a $0.30^{\prime \prime}$ AP ammunition (Figure 3), and its dimensions are shown in Figure 4; its exterior material and interior materials are copper and steel, respectively. A 1/4 scale symmetrical model was constructed, whereby the reflect function was used to present the full bullet figure during analysis; material model 3 (Mat_Plastic_Kinematic) is suited to model isotropic material, therefore was chosen to describe the steel component of the bullet in this study. The mechanical properties of the steel are shown in Table 2. Material model 10 (Mat_Elastic_Plastic_Hydro) allows the modeling of an elastic-plastic hydrodynamic material, and the characteristics of copper are suited to use this model. The mechanical properties of copper are shown in Table 3.

The target plate model was divided into $\mathrm{SiC}$ ceramic and Dyneema fiber (Figure 5). Material model 110 (MAT_Johnson_Holmquist_Ceramics) is useful for modeling ceramics [13], therefore was chosen to describe $\mathrm{SiC}$ ceramic. Mechanical properties of $\mathrm{SiC}$ are shown in Table 4. Dyneema fiber was described using material model 22 (MAT_Composite_Damage), in which an orthotropic material with optional brittle failure for composite can be defined. Mechanical properties of Dyneema are shown in Table 5. The aforementioned material parameter setting is referred to the paper presented by Fawaz et al. [14]. In addition, the "Contact_Eroding_Surface_To_Surface" contact model in LS_DYNA was chosen to describe the dynamic behavior between bullet and $\mathrm{SiC}$ ceramic as well as bullet and

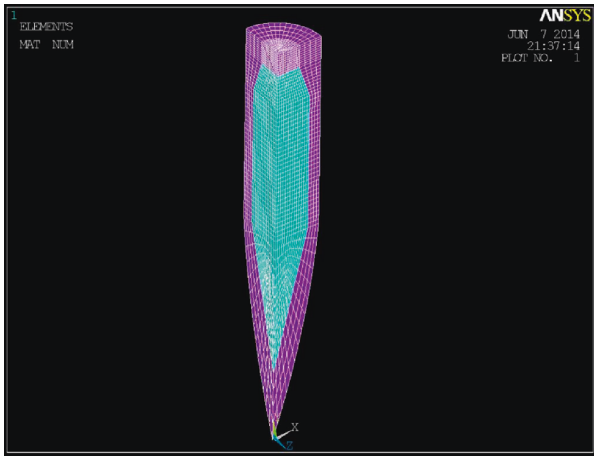

FIgURE 3: $0.30^{\prime \prime}$ AP model.

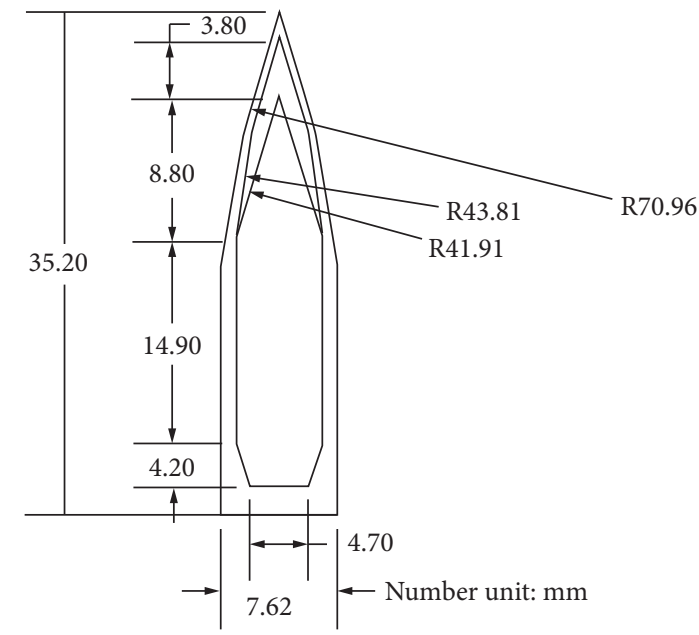

FIgURE 4: $0.30^{\prime \prime}$ AP bullet dimensions.

Table 2: Mechanical properties of material model 3 (Mat_Plastic_Kinematic).

\begin{tabular}{lcccc}
\hline Materials & $\rho\left(\mathrm{g} / \mathrm{cm}^{3}\right)$ & $E($ Mbar $)$ & $v$ & SIGY (Mbar) \\
\hline Steel & 7.89 & 2.02 & 0.3 & 0.0107 \\
\hline
\end{tabular}

TABle 3: Mechanical properties of material model 10 (Mat_Elastic_Plastic_Hydro).

\begin{tabular}{lcccc}
\hline Materials & $\rho\left(\mathrm{g} / \mathrm{cm}^{3}\right)$ & SIGY (Mbar) & EH (Mbar) & $G($ Mbar $)$ \\
\hline $\mathrm{Cu}$ & 8.93 & 0.0012 & 0.00288 & 0.477 \\
\hline
\end{tabular}

Dyneema fiber. After the fiber board was damaged by the bullet, the fiber will generate layer-stripped phenomenon, and the layers will delaminate. Among those "Contact" commands, we chose the "Contact_Tied_Surface_To_Surface" to describe the dynamic behavior among the layers of the Dyneema fiber. In this contact mode, the nodes are stuck together before the fiber board was damaged. When the material of the fiber is subjected to external force, damage will occur in accordance with the failure criterion. The failure criterion is shown as equation (2), controlled by tensile failure stress and shear failure stress. 


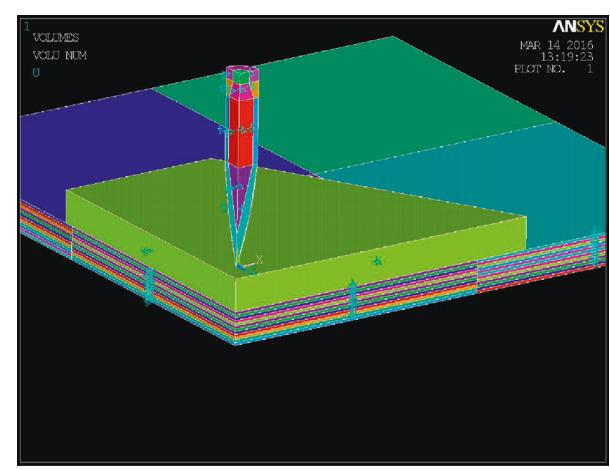

Figure 5: Target plate model.

TABLE 4: Mechanical properties of SiC.

\begin{tabular}{lc}
\hline$\rho$ & $3.1\left(\mathrm{~g} / \mathrm{cm}^{3}\right)$ \\
$G$ & $1.79(\mathrm{Mbar})$ \\
$A$ & $0.96[8,14]$ \\
$B$ & $0.35[8,14]$ \\
$C$ & $0.0[8,14]$ \\
$M$ & $1.0[8,14]$ \\
$N$ & $0.65[8,14]$ \\
$T$ & $0.001275(\mathrm{Mbar})$ \\
$\mathrm{HEL}$ & $0.03738(\mathrm{Mbar})$ \\
$P_{\text {hel }}$ & $0.0174(\mathrm{Mbar})$ \\
$D_{1}$ & $0.48[8,14]$ \\
$D_{2}$ & $0.48[8,14]$ \\
$K_{1}$ & $2.09(\mathrm{Mbar})$ \\
\hline
\end{tabular}

$$
\left(\frac{\left|\sigma_{n}\right|}{\text { NFLS }}\right)^{2}+\left(\frac{\left|\sigma_{s}\right|}{\text { SFLS }}\right)^{2} \geq 1
$$

where NFLS is the tensile failure stress and SFLS is the shear failure stress.

The mesh type used in the simulation model is hexahedron (3D_Solid_164), and the mesh size is as follows (length $*$ width $*$ height):
(a) Steel: $0.021 * 0.021 * 0.04(\mathrm{~cm})$
(b) $\mathrm{Cu}: 0.025 * 0.025 * 0.04(\mathrm{~cm})$
(c) Ceramic: $0.086 * 0.1 * 0.1(\mathrm{~cm})$
(d) Fiber: $0.1 * 0.1 * 0.05(\mathrm{~cm})$

Because only a quarter of the target plate and the bullet model were established, the boundary conditions of the numerical simulation in this study are set as the BOUNDARY_SYMMETRY_FAILURE model to allow the simulation results to map out the remaining three-quarters of the model and avoid unreasonable damage and physical phenomena; the $X$-, $Y$-, and $Z$-axis movement and rotation direction of all nodes on the symmetry axis and plane should be restrained according to the actual situation. The all sides of the back plate were set fixed.

3.4. Comparison of Numerical Simulation and Ballistic Test Results. The ballistic test result in this study was compared with that of numerical simulation analysis. Excluding the construction of material models, the required computational time was 2 hours.

Constructed from a combination of $6 \mathrm{~mm} \mathrm{SiC} \mathrm{ceramic}$ and $6 \mathrm{~mm}$ Dyneema fiber plates, the ceramic composite target plate in this study underwent ballistic experiments and numerical simulation on plate penetration. Through the initial velocity of the bullet and the residual velocity measured after the bullet penetrated the plate, it was estimated that the absorptive energy of the plate was $2,501 \mathrm{~J}$. In addition, assuming the initial velocity of the bullet was the same with the ballistic experiments, the numerical simulation analysis indicated that the residual velocity of the bullet after it penetrated the target plate was $532 \mathrm{~m} / \mathrm{s}$, and the absorptive energy of the target plate was estimated to be $2,558 \mathrm{~J}$. The results indicated a difference of $2.276 \%$ between the numerical simulation analysis and the ballistic experiments (Table 6), which verifies that the LS-DYNA model and parameter settings can be used to simulate and analyze the actual bullet impact phenomenon.

\section{Optimal Thickness Combination of the Ceramic Composite}

4.1. Experimental Design. To effectively lower the time required for material model construction and numerical computation, this study formulated factor combinations to reduce the number of experiments required. The empirical function was then obtained through regression analysis to shorten the simulation time.

Based on previous experiences with ballistic experiments, a composite target plate is deemed to meet the NIJ IV standards if it is $15 \mathrm{~mm}$ thick and the ceramic part is $10 \mathrm{~mm}$ thick. To acquire the ballistic resistance value of the plate, the experiment was designed as a penetration test. Therefore, the thickness of the test plate should not exceed $15 \mathrm{~mm}$, and the thickness of the ceramic plate should not exceed $10 \mathrm{~mm}$.

Based upon face-centered cubic composite design, the thickness of the SiC ceramic plate $\left(h_{c}\right)$ and thickness of the fiber plate $\left(h_{f}\right)$ were used as experimental factors, and the absorptive energy of the target plate $\left(E_{A}\right)$ was the reaction value. The factor combination was formulated using the 2factor-3-level model (Table 7). The unit for $h_{c}$ and $h_{f}$ was $\mathrm{mm}$, and the unit for $E_{A}$ was $\mathrm{J}$.

4.2. Test Results. The bullet mass was assumed to remain constant throughout the experiment, and the initial velocity of the bullet was controlled at $875 \mathrm{~m} / \mathrm{s}$. Through numerical analysis following the construction of material models, the residual velocity of the bullet after penetrating the plate was obtained, wherein $E_{A}$ was calculated based on the dynamic values of the bullet velocity (Table 8 ).

Next, the adaptive quadratic reaction surface method was conducted using $h_{c}$ and $h_{f}$ as operational variables, with $E_{A}$ as the reaction value. This could also be viewed as the energy function (equation (3) whereupon the adaptive function could be obtained following analysis equation (4)): 
TABle 5: Mechanical properties of Dyneema.

\begin{tabular}{lcccccccccc}
\hline$E_{11,22}(\mathrm{GPa})$ & $E_{33}(\mathrm{GPa})$ & $G_{12}(\mathrm{GPa})$ & $G_{23,31}(\mathrm{GPa})$ & $v_{22}$ & $v_{31,32}$ & $X_{t}(\mathrm{GPa})$ & $Y_{t}(\mathrm{GPa})$ & $X_{c}(\mathrm{GPa})$ & $Y_{c}(\mathrm{GPa})$ & $\rho\left(\mathrm{g} / \mathrm{cm}^{3}\right)$ \\
\hline 30.7 & 1.97 & 1.97 & 0.67 & 0.008 & 0.044 & 3.0 & 3.0 & 0.95 & 0.95 & 0.97 \\
\hline
\end{tabular}

$E_{i j}$ : Young' modulus, $G_{i j}$ : shear modulus, $v_{i j}$ : Poisson's ratio, $\rho$ : density, $1-3: x$-, $y$-, and $z$-axis directions of materials, $X_{t}, X_{c}$ : maximum tensile and compressive strength of $x$-axis directions, and $Y_{t}, Y_{c}$ : maximum tensile and compressive strength of $y$-axis directions.

TABLE 6: Comparison of target plate absorptive energy in the numerical simulation and ballistic tests.

\begin{tabular}{lcccc}
\hline \multicolumn{2}{c}{ Ballistic experiments } & \multicolumn{2}{c}{ Numerical simulation } & Target plate absorptive energy difference (\%) \\
$v_{i}(\mathrm{~m} / \mathrm{s})$ & $v_{r}(\mathrm{~m} / \mathrm{s})$ & $v_{i}(\mathrm{~m} / \mathrm{s})$ & $v_{r}(\mathrm{~m} / \mathrm{s})$ & 2.276 \\
\hline 875 & 542 & 875 & 532 & \\
\hline
\end{tabular}

TABle 7: Experimental design.

\begin{tabular}{lcc}
\hline No. & $h_{c}(\mathrm{~mm})$ & $h_{f}(\mathrm{~mm})$ \\
\hline 1 & 5 & 4 \\
2 & 5 & 6 \\
3 & 5 & 8 \\
4 & 6 & 4 \\
5 & 6 & 6 \\
6 & 6 & 8 \\
7 & 7 & 4 \\
8 & 7 & 6 \\
9 & 7 & 8 \\
\hline
\end{tabular}

TABle 8: Test data.

\begin{tabular}{lccccc}
\hline No. & $h_{c}(\mathrm{~mm})$ & $h_{f}(\mathrm{~mm})$ & $v_{i}(\mathrm{~m} / \mathrm{s})$ & $v_{r}(\mathrm{~m} / \mathrm{s})$ & $E_{A}(\mathrm{~J})$ \\
\hline 1 & 5 & 4 & 875 & 768 & 932 \\
2 & 5 & 6 & 875 & 641 & 1,880 \\
3 & 5 & 8 & 875 & 612 & 2,073 \\
4 & 6 & 4 & 875 & 719 & 1,318 \\
5 & 6 & 6 & 875 & 532 & 2,558 \\
6 & 6 & 8 & 875 & 488 & 2,796 \\
7 & 7 & 4 & 875 & 632 & 1,941 \\
8 & 7 & 6 & 875 & 412 & 3,158 \\
9 & 7 & 8 & 875 & 347 & 3,420 \\
\hline
\end{tabular}

$$
\begin{aligned}
E_{A}\left(h_{c}, h_{f}\right)= & k+a h_{c}+b h_{f}+c h_{c} h_{f}+d h_{c}^{2}+e h_{f}^{2} \\
E_{A}\left(h_{c}, h_{f}\right)= & -6276.5+500.6 h_{c}+1512.6 h_{f}+30.7 h_{c} h_{f} \\
& -8.1 h_{c}^{2}-113.6 h_{f}^{2}
\end{aligned}
$$

\subsection{Analysis Results}

4.3.1. Regression Function Limitations. According to equation (3), when the values of both $h_{c}$ and $h_{f}$ were 0 , the value obtained for $E_{A}$ was less than 0 , which was clearly inconsistent with real-world physics. Thus, there were limitations to the application of equation (3).

Because the quadratic coefficients of $h_{c}$ and $h_{f}$ were both smaller than 0 , it was clear that the target plate thickness had maximal influence on $E_{A}$. Therefore, the maximal operational variable of the energy function could be acquired by subjecting $h_{c}$ and $h_{f}$ in equation (2) to differentiation (equation (5)).

$$
\left\{\begin{array} { l } 
{ \frac { \partial E _ { A } } { \partial h _ { c } } = 0 \longrightarrow a + c h _ { f } + 2 d h _ { c } = 0 } \\
{ \frac { \partial E _ { A } } { \partial h _ { f } } = 0 \longrightarrow b + c h _ { c } + 2 e h _ { f } = 0 }
\end{array} \Longrightarrow \left\{\begin{array}{l}
\left(h_{c}\right)_{\max }=\frac{-2 a e+b c}{4 d e-c^{2}} \\
\left(h_{f}\right)_{\max }=\frac{-2 b d+a c}{4 d e-c^{2}}
\end{array}\right.\right.
$$

4.3.2. Minimal Target Plate Thickness. Equation (6) shows $h_{c}$ being arranged in a series of descending power. The target plate thickness $\left(\theta=h_{c}+h_{f}\right)$ was used as the analysis parameter and was held constant. When $h_{f}$ was substituted with target plate thickness $(\theta)$ and $h_{c}, E_{A}$ was a function of $\theta$ and $h_{c}$ :

$$
\begin{aligned}
E_{A}\left(\theta, h_{c}\right)= & (-c+d+e) h_{c}^{2}+[(a-b)+\theta(c-2 e)] h_{c} \\
& +\left(k+b \theta+e \theta^{2}\right) \\
= & P_{1} h_{c}^{2}+Q_{1}(\theta) h_{c}+R_{1}(\theta) \\
= & P_{1}\left(h_{c}+\frac{Q_{1}(\theta)}{2 P_{1}}\right)^{2}+\left(R_{1}(\theta)-\frac{Q_{1}^{2}(\theta)}{4 P_{1}}\right) \\
\Longrightarrow & R_{1}(\theta)-\frac{Q_{1}^{2}(\theta)}{4 P_{1}} \geq E_{A}\left(\theta, h_{c}\right) \\
\Longrightarrow & {\left[E_{A}\left(\theta, h_{c}\right)\right]_{\max }=R_{1}(\theta)-\frac{Q_{1}^{2}(\theta)}{4 P_{1}} }
\end{aligned}
$$

$$
P<0
$$

According to equation (5), $E_{A}$ had a maximal value when $\theta$ remained a fixed value. In other words, when $E_{A}$ was a known value, the minimal target plate thickness $\left(\theta_{\min }\right)$ as well as the optimal thickness combination of the $\mathrm{SiC}$ ceramic plate and Dyneema fiber plate could be inversely calculated. Thus, when the value of $E_{A}$ was 0 , the minimal value of $\theta$ and $h_{c}$ could be inversely calculated according to the following equation: 


$$
\begin{aligned}
\theta_{\min }= & {\left[\left(h_{c}\right)_{\max }+\left(h_{f}\right)_{\max }\right]-\left(\frac{2}{4 d e-c^{2}}\right) } \\
& \cdot \sqrt{(-c+d+e)\left[\left(a^{2} e+b^{2} d-a b c\right)-\left(4 d e-c^{2}\right) k\right]} \\
\left(h_{c}\right)_{\min }= & \left(h_{c}\right)_{\max }+\left(\frac{c-2 e}{4 d e-c^{2}}\right) \\
& \cdot \sqrt{\frac{\left(a^{2} e+b^{2} d-a b c\right)-\left(4 d e-c^{2}\right) k}{(-c+d+e)}} .
\end{aligned}
$$

4.3.3. Minimal Areal Density. This study sought to design a light-weight ballistic-resistant target plate under the NIJ IV standard. Hence, areal density $(\Psi)$ was used as the analysis parameter (equation (8)):

$$
\begin{gathered}
\Psi\left(h_{c}, h_{f}\right)=\rho_{c} h_{c}+\rho_{f} h_{f} \\
\Longrightarrow \frac{\Psi}{\rho_{f}}=\frac{\rho_{c}}{\rho_{f}} h_{c}+h_{f} \\
\Longrightarrow \alpha=\beta h_{c}+h_{f},
\end{gathered}
$$

where the volume densities of SiC ceramic $\left(\rho_{c}\right)$ and Dyneema fiber were $3.1 \times 10^{-3}\left(\mathrm{~g} / \mathrm{mm}^{3}\right)$ and $0.97 \times 10^{-3}\left(\mathrm{~g} / \mathrm{mm}^{3}\right)$, respectively; $\alpha$ is the ratio of $\Psi$ over $\rho_{f}$, and $\beta$ is the ratio of $\rho_{c}$ over $\rho_{f}$.

Equation (9) shows $h_{c}$ being arranged in a series of descending power. The value of target areal density $\left(\Psi=\rho_{c} h_{c}+\rho_{f} h_{f}\right)$ remained constant, and $h_{f}$ was substituted with $\Psi$ and $h_{c}$. $E_{A}$ was thus a function of $\alpha$ and $h_{c}$ :

$$
\begin{aligned}
E_{A}\left(\alpha, h_{c}\right)= & \left(-c \beta+d+e \beta^{2}\right) h_{c}^{2}+[(a-b \beta)+\alpha(c-2 e \beta)] h_{c} \\
& +\left(k+b \alpha+e \alpha^{2}\right) \\
= & P_{2} h_{c}^{2}+Q_{2}(\alpha) h_{c}+R_{2}(\alpha) \\
= & P_{2}\left(h_{c}+\frac{Q_{2}(\alpha)}{2 P_{2}}\right)^{2}+\left(R_{2}(\alpha)-\frac{Q_{2}^{2}(\alpha)}{4 P_{2}}\right) \\
\Longrightarrow & R_{2}(\alpha)-\frac{Q_{2}^{2}(\alpha)}{4 P_{2}} \geq E_{A}\left(\alpha, h_{c}\right) \\
\Longrightarrow & {\left[E_{A}\left(\alpha, h_{c}\right)\right]_{\max }=R_{2}(\alpha)-\frac{Q_{2}^{2}(\alpha)}{4 P_{2}} } \\
P_{2}< & 0 .
\end{aligned}
$$

According to equation (9), $E_{A}$ had a maximal value when the value of $\Psi$ was held constant. In other words, minimal $\Psi$ and the optimal combined thickness of $\mathrm{SiC}$ ceramic and Dyneema fiber could be inversely calculated when $E_{A}$ was a known value. Therefore, when the value of $E_{A}$ was 0 , the minimal value of $\Psi$ and $h_{c}$ could be inversely calculated as follows:

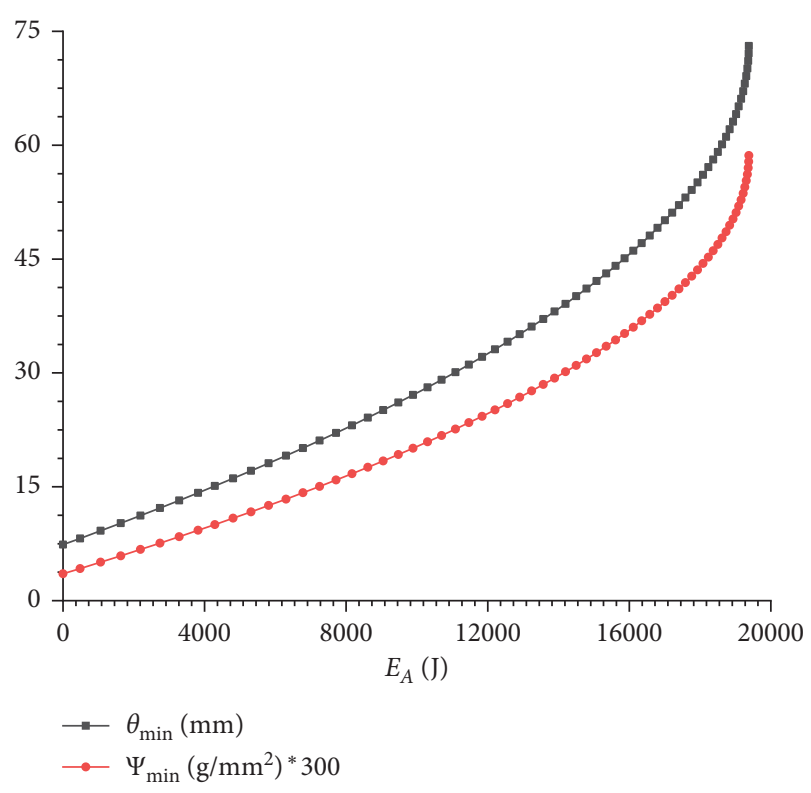

FIGURE 6: Absorptive energy corresponded to minimization of thickness and areal density of the target.

$$
\begin{aligned}
\Psi_{\min }= & {\left[\rho_{c}\left(h_{c}\right)_{\max }+\rho_{f}\left(h_{f}\right)_{\max }\right]-\left(\frac{2 \rho_{f}^{2}}{4 d e-c^{2}}\right) } \\
& \cdot \sqrt{\left(-c \beta+d+e \beta^{2}\right)\left[\left(a^{2} e+b^{2} d-a b c\right)-\left(4 d e-c^{2}\right) k\right]} \\
\left(h_{c}\right)_{\min }= & \left(h_{c}\right)_{\max }+\left(\frac{c-2 e \beta}{4 d e-c^{2}}\right) \\
& \cdot \sqrt{\frac{\left(a^{2} e+b^{2} d-a b c\right)-\left(4 d e-c^{2}\right) k}{\left(-c \beta+d+e \beta^{2}\right)}} .
\end{aligned}
$$

4.3.4. Optimal Combination. To completely absorb the kinetic energy of the bullet using the $\mathrm{SiC}$ ceramic and Dyneema fiber composite target plate, the optimal combination of $h_{c}$ and $h_{f}$ could be obtained through the aforementioned numerical analysis (Figures 6 and 7).

\subsection{Experimental Verification}

4.4.1. Energy Function Verification. The energy function of the ballistic-resistant target plate was obtained through experimentally designed regression analysis, wherein the target plate thickness was maintained between $9 \mathrm{~mm}$ and $15 \mathrm{~mm}$. Hence, four sets of target plate thickness combinations were chosen from the range and were subjected to numerical simulations to verify the energy function (Table 9). The results indicated that the optimal combination featuring minimal target plate thickness $\left(\theta_{\min }\right)$ which corresponded to the energy function and satisfied the NIJ IV standards was consistent with that obtained from the numerical simulation analysis. 


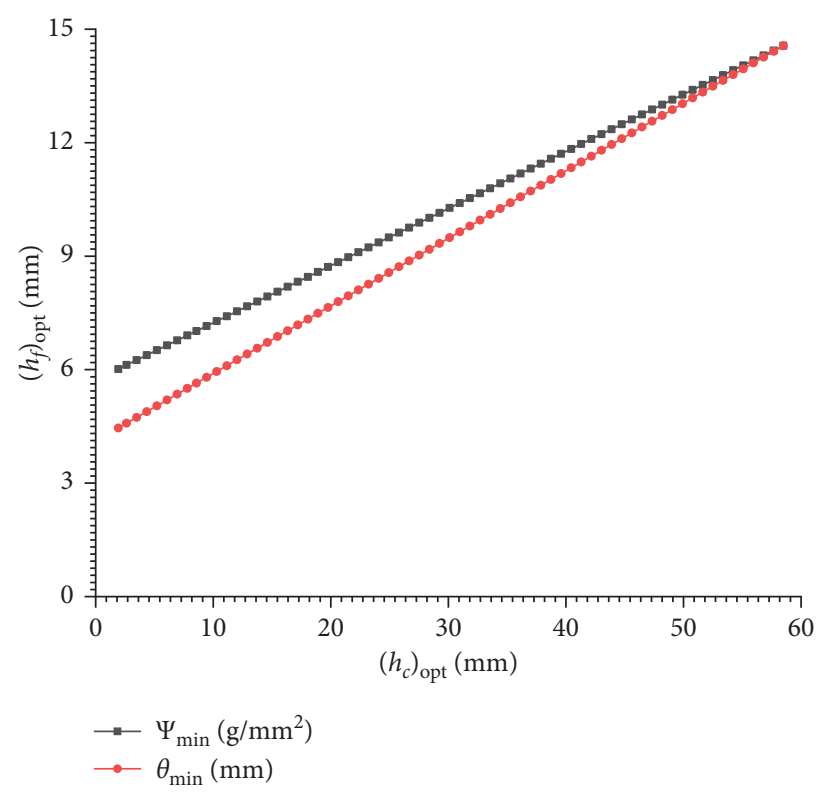

Figure 7: Optimal thickness combination of the composite materials.

TABLe 9: Verification of the energy function.

\begin{tabular}{lccccccc}
\hline & \multicolumn{2}{c}{ Energy function } & \multicolumn{3}{c}{ Numerical simulation } & \multicolumn{2}{c}{ Difference (\%) } \\
$\theta_{\min } \mathrm{mm}$ & $\left(h_{c}\right)_{\text {opt }} \mathrm{mm}$ & $\left(h_{f}\right)_{\text {opt }} \mathrm{mm}$ & $\left(E_{A}\right)_{1} \mathrm{~J}$ & $v_{i} \mathrm{~m} / \mathrm{s}$ & $v_{r} \mathrm{~m} / \mathrm{s}$ & $\left(E_{A}\right)_{2} \mathrm{~J}$ & $\left(\left(E_{A}\right)_{1} /\left(E_{A}\right)_{2}\right)-1$ \\
\hline 9.0 & 4.2949 & 4.7051 & 946.5579 & 875.0 & 762.0 & 980.3993 & -3.451 \\
12.5 & 7.2564 & 5.2436 & $2,905.6687$ & 875.0 & 467.0 & 2901.9408 & 0.13 \\
14.7 & 9.1179 & 5.5821 & $4,080.7837$ & 875.0 & 0.0 & $4,057.8125$ & 0.57 \\
15.0 & 9.3717 & 5.6283 & $4,237.6600$ & 875.0 & 0.0 & $4,057.8125$ & 4.43 \\
\hline
\end{tabular}

TABLE 10: Verification of the optimal combination.

\begin{tabular}{lcccccc}
\hline & \multicolumn{2}{c}{ Energy function } & \multicolumn{4}{c}{ Numerical simulation } \\
$\Psi_{\min }$ & $\begin{array}{c}\left(h_{c}\right)_{\text {opt }} \\
\mathrm{mm}\end{array}$ & $\begin{array}{c}\left(h_{f}\right)_{\text {opt }} \\
\mathrm{mm}\end{array}$ & $\left(E_{A}\right)_{1} \mathrm{~J}$ & $v_{i} \mathrm{~m} / \mathrm{s}$ & $v_{r}$ & $\left(E_{A}\right)_{2} \mathrm{~J}$ \\
$\mathrm{~g} / \mathrm{mm}^{2}$ & 8.1940 & 6.9637 & $4,057.8$ & 875.00 & 0.00 & $4,057.8$ \\
\hline 0.032 & 8.196 &
\end{tabular}

4.4.2. Verifying the Optimal Combination. This study aimed to develop light-weight ballistic-resistant target plates while conforming to NIJ IV standards. Therefore, the energy function was verified using the optimal combination with minimal areal density $\left(\Psi_{\text {min }}\right)$ (Table 10$)$. The results indicated that, in the estimation of the absorptive energy of the target plate, the energy function fits the numerical simulation analysis.

\section{Conclusion}

This study explored the relationship between the $\mathrm{SiC}$ ceramic and Dyneema fiber layers in a composite target plate and its ballistic resistance. To achieve a light-weight target plate that conforms to NIJ IV standards, an analysis of the minimal areal density was conducted to obtain the optimal $\mathrm{SiC}$ ceramic-Dyneema fiber thickness combination.

This study analyzed the ballistic resistance of the target plate using energy modes. To substantially reduce the experimental costs, ANSYS/LS-DYNA software was used to conduct finite element method numerical simulations and to obtain the correlation function of the target plate ballistic resistance. The ballistic resistance, which is also the function of the absorptive energy of the target plate, was obtained using simulations and regression analysis. From the numerical analysis results, $8.1940 \mathrm{~mm} \mathrm{SiC} \mathrm{ceramic} \mathrm{and}$ $6.9637 \mathrm{~mm}$ Dyneema fiber were determined to be the optimal thickness combination for a composite that features a minimal areal density and which conforms to NIJ IV standards. The combination was found to be consistent with the numerical simulation analysis results.

\section{Data Availability}

All data used to support the findings of this study are included in the tables within the article.

\section{Conflicts of Interest}

The authors declare that they have no conflicts of interest.

\section{References}

[1] P. Riou, C. Denoual, and C. E. Cottenot, "Visualization of the damage evolution in impacted silicon carbide ceramics," International Journal of Impact Engineering, vol. 21, no. No. 4, pp. 225-235, 1998. 
[2] M. L. Wilkins, "Mechanics of penetration and perforation," International Journal of Engineering Science, vol. 16, no. 11, pp. 793-807, 1978.

[3] H. L. Hou, X. Zhu, and Y. L. Kan, "The advance of ballistic performance of light ceramic composite armour under the impact of projectile," Acta Armament Arii, vol. 29, no. 2, 2008.

[4] D. Sherman, "Impact failure mechanisms in alumina tiles on finite thickness support and the effect of confinement," International Journal of Impact Engineering, vol. 24, no. 3, pp. 313-328, 2000.

[5] Z. H. Du, G. B. Wang, P. H. Shen, and G. Z. Zhao, "Analytical model of ceramic/kevlar composite amour defensible performance," Journal of Ballistics, vol. 15, no. 2, 2003.

[6] S. T. Li, S. Y. Tian, T. Zhong, B. R. Zhao, and X. Wu, "Effect of fiber-layer with different thickness on ballistic behavior of ceramic composite armor," Ordnance Material Science and Engineering, vol. 31, no. 6, 2008.

[7] Y. L. Hsieh, K. C. Lee, W. Y. Chang, and M. H. Tseng, "Impact destruction of bullet proof ceramic compound material," Journal of the Hwa Gang Textile, vol. 15, no. 4, 2008.

[8] B. Chen, X. R. Luo, and S. Y. Zeng, "Simulation study on ceramic/mild steel targets penetrated by APP," Journal of Ballistics, vol. 21, no. 1, 2009.

[9] E. Ömer, I. Birhan, Ç. Adem, and K. Fuat, "Prediction of damage factor in end milling of glass fibre reinforced plastic composites using artificial neural network," Applied Composite Materials, vol. 20, pp. 517-536, 2012.

[10] Ö. Erkan, M. Demetgül, B. Ișik, and İ. N. Tansel, "Selection of optimal machining conditions for the composite materials by using Taguchi and GONNs," Measurement, vol. 48, pp. 306313,2014

[11] S. Gökhan and E. Ömer, "Cutting tool geometry in the drilling of CFRP composite plates and Taguchi optimisation of the cutting parameters affecting delamination," Sigma Journal of Engineering and Natural Sciences, vol. 36, no. 3, pp. 619-628, 2018.

[12] B. C. Shen, Penetration resistance of steel fiber reinforced concrete structures, Ph.D. thesis, School of Defense Science, Chung Cheng Institute of Technology National Defense University, Taoyuan, Taiwan, 2007.

[13] S. Duane, K. B. Cronin, K. Christian, M. Grant, and B. Todd, "Implementation and validation of the Johnson-Holmquist ceramic material model in LS-DYNA," in Proceedings of the 4th European LS-DYNA Users Conference, Ulm, Germany, May 2003.

[14] Z. Fawaz, W. Zheng, and K. Behdinan, "Numerical simulation of normal and oblique ballistic impact on ceramic composite armours," Composite Structures, vol. 63, no. 3-4, pp. 387-395, 2004. 


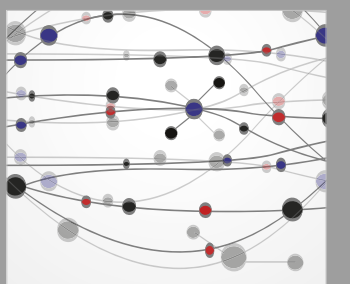

The Scientific World Journal
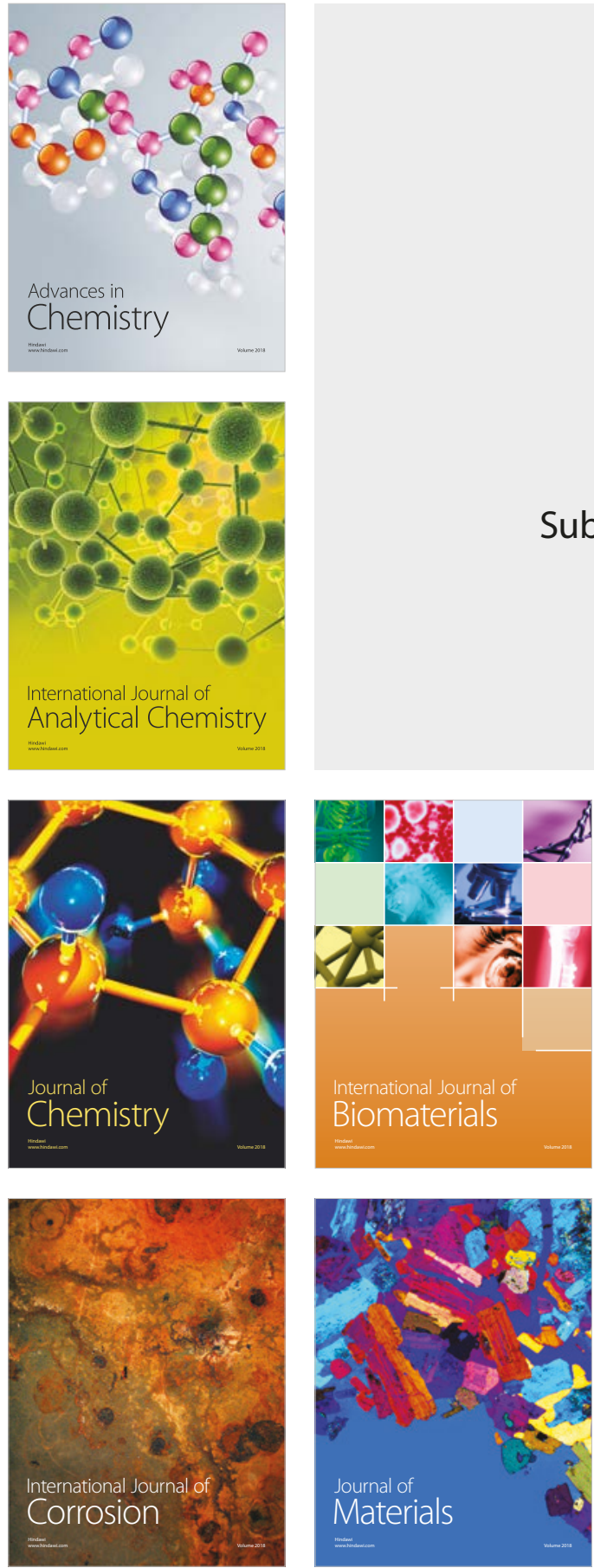

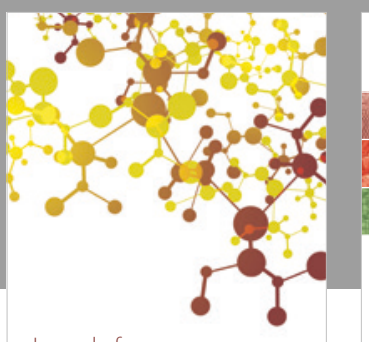

Journal of

Applied Chemistry
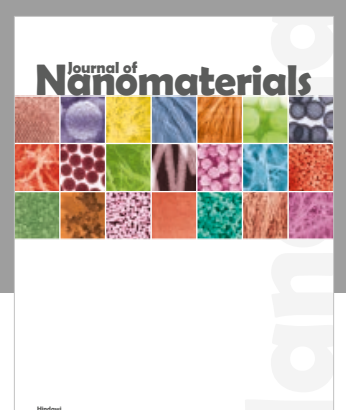

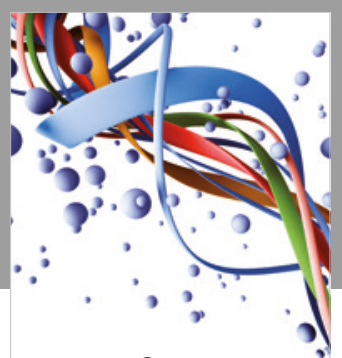

Scientifica

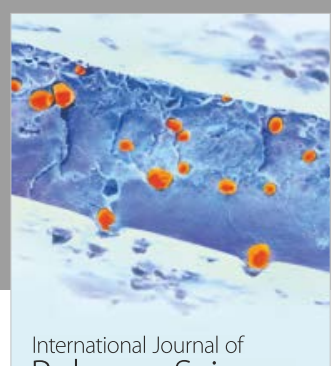

Polymer Science

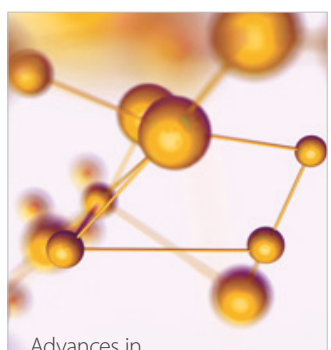

Physical Chemistry
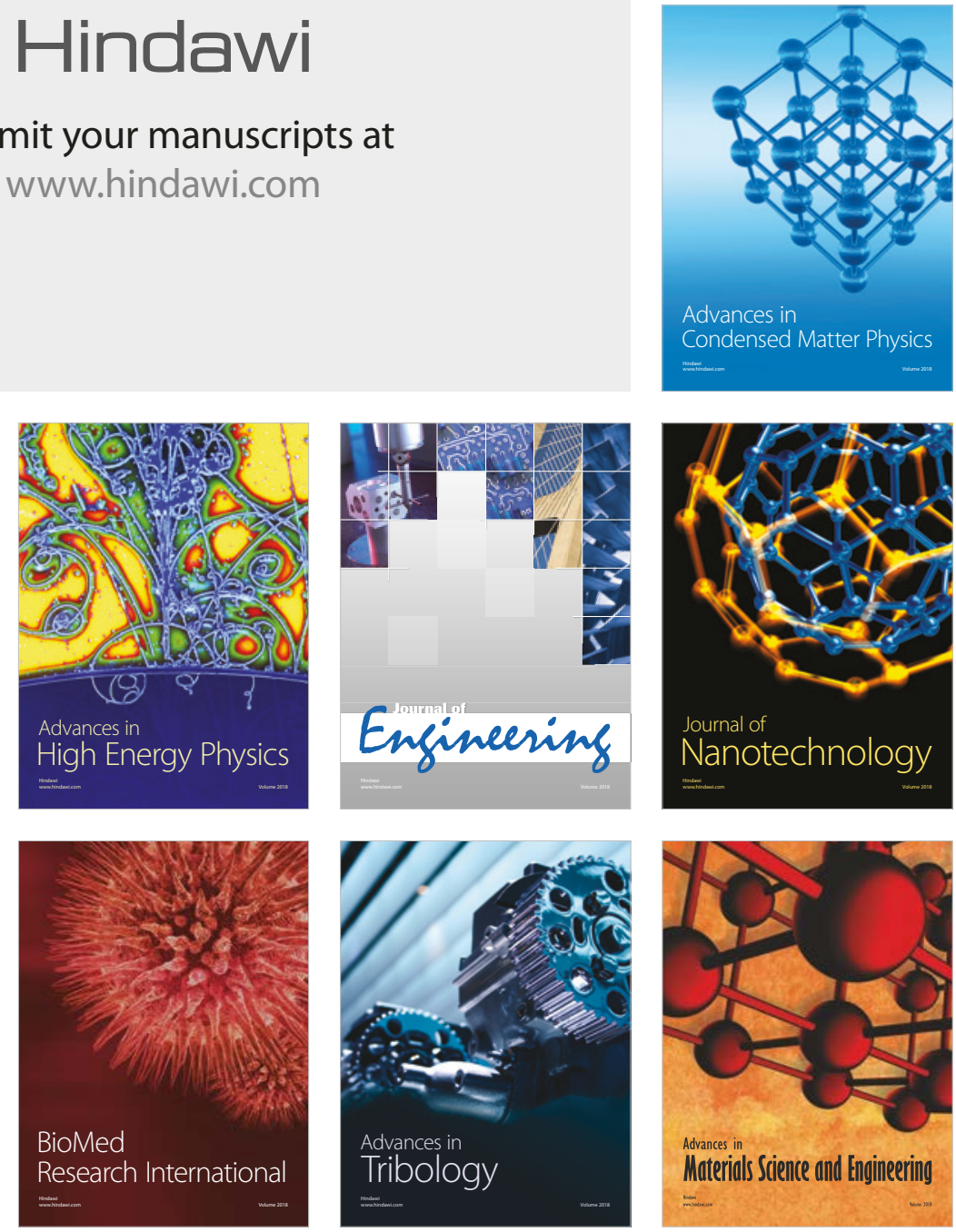